\title{
DESCENT TRAJECTORY RECOVERY OF CHANG'E-4 LANDER BASED ON DESCENT IMAGES
}

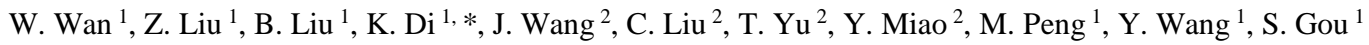 \\ ${ }^{1}$ State Key Laboratory of Remote Sensing Science, Institute of Remote Sensing and Digital Earth, Chinese Academy of Sciences, \\ Beijing, China - (wanwh, liuzq, liubin, dikc, pengman, wangyx716, gousheng)@ radi.ac.cn \\ ${ }^{2}$ Beijing Aerospace Control Center, Beijing, China -(15210106156@139.com, ckliu2005@126.com, i_am_yty@sina.com, \\ miaoyi_2008@126.com)
}

\section{Commission III, ICWG III/II}

KEY WORDS: Chang'e-4 mission, descent image, descent trajectory, bundle adjustment, self-calibration

\begin{abstract}
Chang'e-4 lander, carrying Yutu-2 rover, was successfully landed on the far side of lunar surface in Von Kármán crater inside the South Pole-Aitken basin on January 3rd, 2019. The descent images, captured by the descent camera mounted on the lander, captured the sequential descent images and recorded the scene changes during the entry, descent and landing (EDL) process. This paper proposed a bundle adjustment based geometric processing method for descent and landing trajectory recovery using descent images. A frame camera based self-calibration model was introduced for high precision estimation of interior and exterior parameters of descent images simultaneously in a least squares manners. Evenly distributed GCPs were selected from the landing area in a digital orthophoto map generated from LROC NAC images and SLDEM2015. The experimental results demonstrated the effectiveness of the proposed method in Chang'e-4 descent trajectory recovery.
\end{abstract}

\section{INTRODUCTION}

Chang'e-4 probe has successfully landed on the far side of the moon in Von Kármán crater inside the South Pole-Aitken basin on Jan. $3^{\text {rd }}, 2019$. With the support of Queqiao Relay satellite, the carried rover Yutu-2 was released from the lander and started the three-month expected lunar surface exploration (Di et al., 2019). This mission was the first success soft landing of human spacecraft on the far side of the moon, which retained the original distribution of crater formed by external impact and had pure electromagnetic environment. More discoveries about lunar evolution are expected to find through the mission.

Chang'e-4 mission had installed nine scientific instruments for exploration tasks (Jia, et al., 2018). Chang'e-4 lander carried four scientific instruments to perform lunar in-situ exploration, including Descent Camera, Terrain Camera, Low Frequency Spectrometer, and Lunar Lander Neutron and Dosimetry. Other four scientific instruments were carried by Yutu-2 for lunar patrol exploration, including Panoramic Camera, Lunar Penetrating Radar, Visible and Near-Infrared Imaging Spectrometer, and Advanced Small Analyzer for Neutrals. The last one, Low Frequency Explorer, was installed on the relay satellite. The Descent Camera, which mounted on the bottom of the lander, captured the sequential decent images and recorded the scene changes during the entry, descent and landing (EDL) process. Thus, the valuable information of EDL process from sequential descent images could be used to precisely recover the descending and landing trajectory of the lander.

\section{DESCENT IMAGE DATA}

The EDL process consisted of seven stages, which were stages of descent preparation, main deceleration, rapid adjustment, approaching, hovering, obstacle avoidance, and slow descent. Compared with Chang'e-3 mission, the designed landing track was more vertical for the consideration of safety landing in the far side of moon, which contained more steep craters. The EDL process started with work of descent camera at the height of $15 \mathrm{~km}$. It took about $687 \mathrm{~s}$ for the soft landing to the surface of Von Kármán crater. During the process, the optical rough obstacle avoidance and Lidar based obstacle avoidance were applied at the height of $2 \mathrm{~km}$ and $100 \mathrm{~m}$ respectively.

Similar to that of Chang'e-3 lander, the field of view of the downward-looking descent camera is $45.4^{\circ}$ and the image size is $1024 \times 1024$ pixels (Wan et al., 2014; Liu et al., 2015). Due to the limitation of telemetry bandwidth, only 59 selected descent images compressed with a ratio of 1:64 were received for quick view. After the separation of lander and rover, about 5300 descent images compressed with a ratio of 1:8 were transferred through high speed wireless channel. Figure 1 shows the 1:64 compression image and 1:8 compression image.

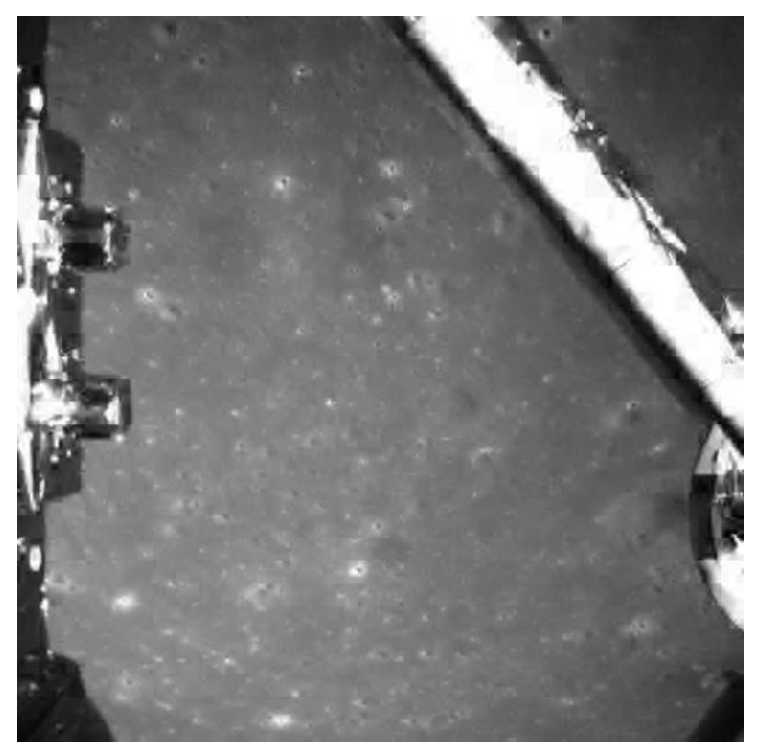

\footnotetext{
* Corresponding author.
} 
(a)The first descent image compressed with 1:64

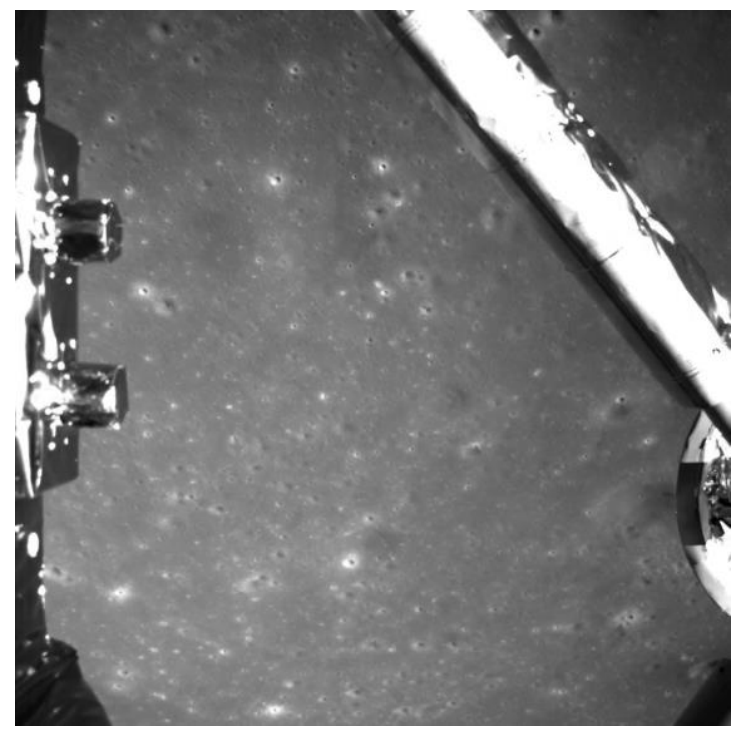

(b)Corresponding descent image compressed with 1:8

Figure 1. The descent images of Chang'e-4 mission

\section{METHODOLOGY}

The recovery of descent trajectory was based on the geometric constraints formed by sequential descent images. In order to construct the image network, the Scale-Invariant Feature Transform (SIFT) was employed for feature extraction and matching between sequential images. Then, the matched points were used to construct the image network as tie points. Due to the lack of accurate interior orientation parameters (IOPs) of the descent camera, the self-calibration model based bundle adjustment was applied to the motion estimation of the sequential images. Even distributed Ground Control Points (GCPs) were selected manually from LRO image and SLDEM for geo-registration and accumulated error elimination of the descent trajectory.

\subsection{Feature extraction and matching}

The feature extraction and matching were the foundation of the image network construction. During the EDL process, with the changes of lander's position and orientation, distortions of scale, rotation and tilt were produced between descent images. SIFT, known for its good performance in images of scaled, rotated and partial tilted, was used to extract the SIFT features in descent images. In order to obtain strong geometric relationship, matching of SIFT features were not only conducted in adjacent images, but also in multi-sequential images. More matching results in multi images were beneficial to construct robust image network and provide more geometric constraint for precious motion estimation.

Fundamental matrix based RANSAC was also applied to outlier detection. Matched results in multi-sequential images were used for robust fundamental matrix estimation by giving higher weights. Those matched results, which did not satisfy the constraint formed by fundamental matrix, would be eliminated through the specified threshold judgement.

\subsection{Self-calibration model}

The trajectory recovery was based on the geometric constraint formed by the sequential descent images. Geometric imaging of these frame perspective images could be formulated as collinear equation, which is described as follow:

$$
\left\{\begin{array}{l}
x=x^{\prime}+x_{0}=f_{x} \frac{X^{\prime}}{-Z^{\prime}}+x_{0} \\
y=y^{\prime}+y_{0}=f_{y} \frac{Y^{\prime}}{-Z^{\prime}}+y_{0}
\end{array}\right.
$$

, where $x^{\prime}, y^{\prime}$ are the coordinates of the object point $\mathbf{P}$ in cameraplane system, $f_{x}, f_{y}$ are the focal length of camera, $x_{0}, y_{0}$ are the image coordinates of principle point, $X^{\prime}, Y^{\prime}, Z^{\prime}$ are the camera space coordinates of $\mathbf{P}$. Moreover, given the exterior parameters of the camera in reference coordinate system, the transformation between coordinate system of reference and camera space can be formulated as follow:

$$
\left[\begin{array}{c}
X^{\prime} \\
Y^{\prime} \\
Z^{\prime}
\end{array}\right]=\mathbf{R}^{-1} \cdot\left[\begin{array}{c}
X-X_{s} \\
Y-Y_{s} \\
Z-Z_{s}
\end{array}\right]=\left[\begin{array}{lll}
a_{1} & b_{1} & c_{1} \\
a_{2} & b_{2} & c_{2} \\
a_{3} & b_{3} & c_{3}
\end{array}\right] \cdot\left[\begin{array}{c}
X-X_{s} \\
Y-Y_{s} \\
Z-Z_{s}
\end{array}\right]
$$

, where $X_{s}, Y_{s}, Z_{s}$ and $X, Y, Z$ are the coordinates of camera and point $\mathbf{P}$ in reference coordinate system respectively, $a_{1}, a_{2}, a_{3}$, $b_{1}, b_{2}, b_{3}, c_{1}, c_{2}, c_{3}$ are the elements of camera rotation matrix.

Taking into account lens distortion, the pin-hole based distortion model was employed for high precision process, which was described as follow:

$\left\{\begin{array}{l}x_{d}^{\prime}=x^{\prime}\left(1+k_{1} r^{2}+k_{2} r^{4}+k_{3} r^{6}\right)+2 p_{1} x^{\prime} y^{\prime}+p_{2}\left(r^{2}+2 x^{2}\right) \\ y_{d}^{\prime}=y^{\prime}\left(1+k_{1} r^{2}+k_{2} r^{4}+k_{3} r^{6}\right)+p_{1}\left(r^{2}+2 y^{2}\right)+2 p_{2} x^{\prime} y^{\prime}\end{array}\right.$

,where $r^{2}=x^{2}+y^{\prime 2}, k_{1}, \quad k_{2}, \quad k_{3}$ were radial distortion coefficients, $p_{1}, p_{2}$ were tangential distortion coefficients. By combination of formula (1) and (3), the formula of the projection from object point $\mathbf{P}$ into the distorted image plane can be expressed as following:

$$
\left\{\begin{array}{l}
x_{d}=x_{d}^{\prime}+x_{0} \\
y_{d}=y_{d}^{\prime}+y_{0}
\end{array}\right.
$$

In general, precise distortion correction requires more valid distortion coefficients of the calibration model, which need to input more valid control points in calibration process. Higher order calibration model may not work well if input insufficient control points. Thus, the order selection of calibration model depends on the quality and distribution of control points.

\subsection{Motion estimation}

Sufficient tie points of the image network could provide necessary constrains to solve the exterior orientation parameters (EOPs) by using the bundle adjustment based model. The solution of $3 \mathrm{D}$ position and orientation of the images was obtained by means of iterative calculation under least square principle.

However, we only got the results of EOPs in local coordinate system without input of reference geo-information. Furthermore, drift errors of EOPs in the sequential images always existed, especially in the edge images of the image networks. Thus, the ground control points were crucial for geo-registration and drift error elimination of image network. In this paper, the GCPs for 
descent trajectory recovery were selected from the LRO seamless DOM produced by the research group of Di (Di et al, 2018). Meanwhile, the height value of the GCPs were identified from the SLDEM-2015. Since the large quality of descent images, the GCPs were selected in key frames, which were judged by the overlapping ratio between sub-sequential of images.

\section{EXPERIMENTAL RESULTS}

Before Chang'e-4 lander was launched, the seamless DOMs of main and backup planned landing area were generated by subarea block bundle adjustment with rational function model and whole area bundle adjustment with thin plate spline model. Both of the DOMs used SLDEM 2015 as elevation reference. According to the lander localization result proposed by Di et al (2018), the landing point is determined to be $\left(177.588^{\circ} \mathrm{E}\right.$, $45.457^{\circ} \mathrm{S}$ ), where is covered by main planned area. Figure 2 shows the DOM \& DEM of Chang'e-4 landing area and the location of Chang'e-4 lander marked with green point. The resolution of DOM was $0.75 \mathrm{~m}$ and that of DEM was $41.5 \mathrm{~m}$. Geographic range of the two productions was from $176.4^{\circ} \mathrm{E}$ to $178.8^{\circ} \mathrm{E}$ and from $45^{\circ} \mathrm{S}$ to $46^{\circ} \mathrm{S}$.

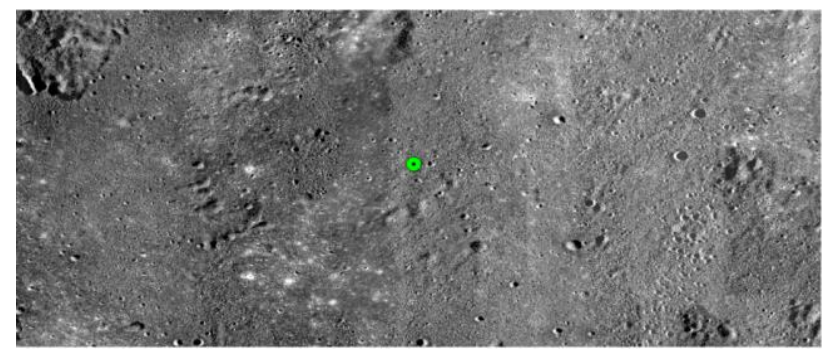

(a) The DOM of Chang'e-4 landing area

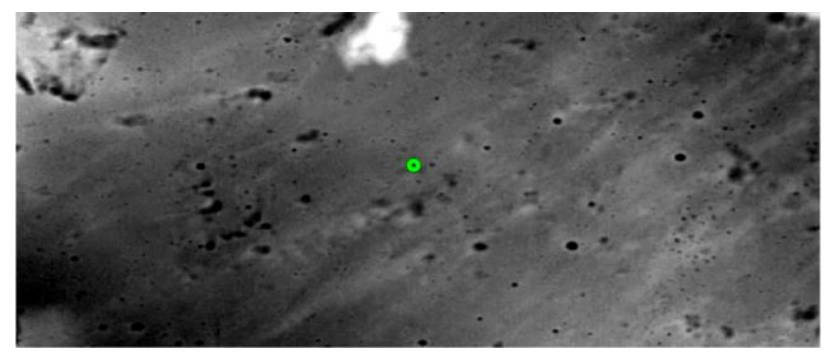

(b) The DEM of Chang'e-4 landing area

Figure 2. The DOM \& DEM of Chang'e-4 landing area and the location of Chang'e-4 lander

During the Chang'e-4 mission, the ground control station received more than 5300 1:8 compressed image at 10fps. In order to save processing time and satisfy the requirement of overlapping ratio between adjacent descent images, we chose 164 descent images from the stage of rapid adjustment. Figure 3 shows a sub-sequential descent image in the stage of hovering.

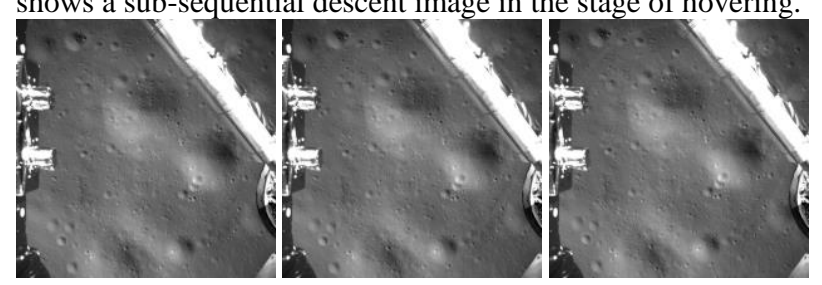

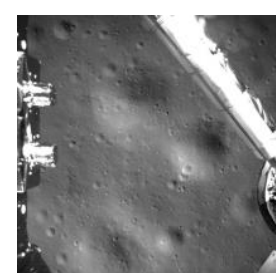
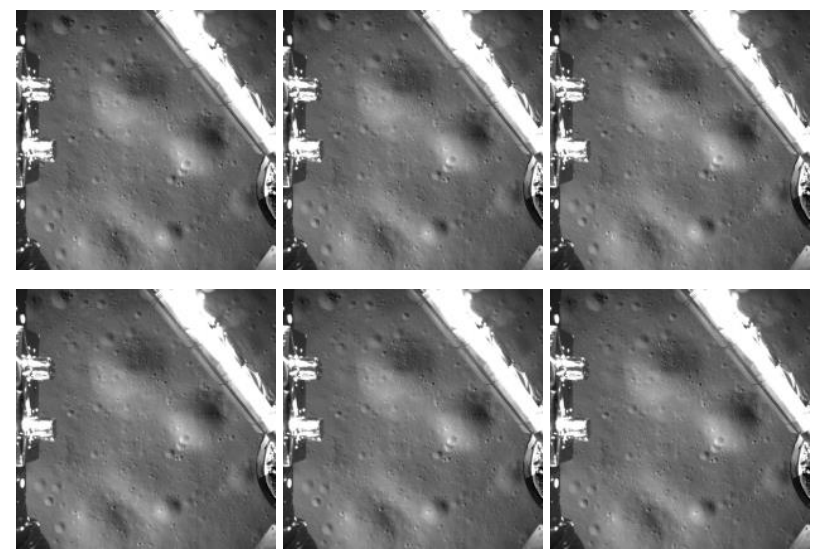

Figure 3. The sub-sequential of descent images

The interval time of these images were mainly 1s. Feature extraction and matching were implemented with SIFT not only between adjacent images, but also in sequential images. Figure 4 shows the matching results between $1^{\text {st }}$ image and $2^{\text {nd }}, 3^{\text {rd }}, 4^{\text {th }}$ image of the selected image sequence.

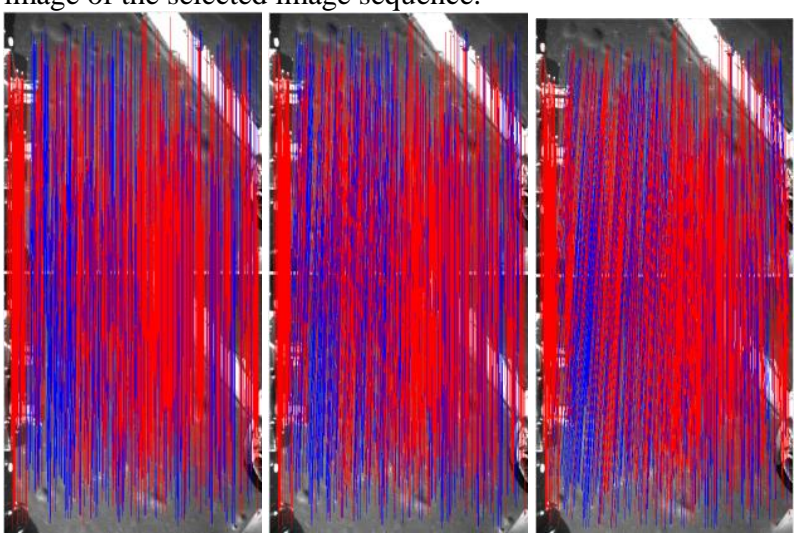

Figure 4 . The matching results between sequential images

In this experiment, GCPs were selected from LRO and SLDEM data for geo-registration and drift error elimination. Since the coordinates of GCPs from the data were geodetic coordinates, they should be transformed into Cartesian coordinates. The transformation formula is show as below:

$$
\left[\begin{array}{l}
X \\
Y \\
Z
\end{array}\right]=\left[\begin{array}{c}
(N+H) \cos B \cos L \\
(N+H) \cos B \sin L \\
{\left[N\left(1-e^{2}\right)+H\right] \sin B}
\end{array}\right]
$$

, where $H$ is the geodetic height, $B$ and $L$ are the latitude and longitude respectively, $e$ is the oblateness, which can be calculated with length of long axis $a$ and short axis $b$ as

$$
e^{2}=\frac{a^{2}-b^{2}}{a^{2}}
$$

.$N$ is the radius of prime vertical, which can be calculated as

$$
N=\frac{a}{\sqrt{1-e^{2} \sin ^{2} B}}
$$

In Moon2000 Geodetic Coordinate System, $a$ and $b$ are set to $1737400 \mathrm{~m}$ equally. So the coordinate transformation formula used in the paper is shown as

$$
\left[\begin{array}{l}
X \\
Y \\
Z
\end{array}\right]=\left[\begin{array}{c}
(R+H) \cos B \cos L \\
(R+H) \cos B \sin L \\
(R+H) \sin B
\end{array}\right]
$$


, where $R$ is $1737400 \mathrm{~m}$.

We selected 12 GCPs from LRO DOM and SLDEM manually for self-calibration based motion estimation. Figure 5 shows the distribution of GCPs.

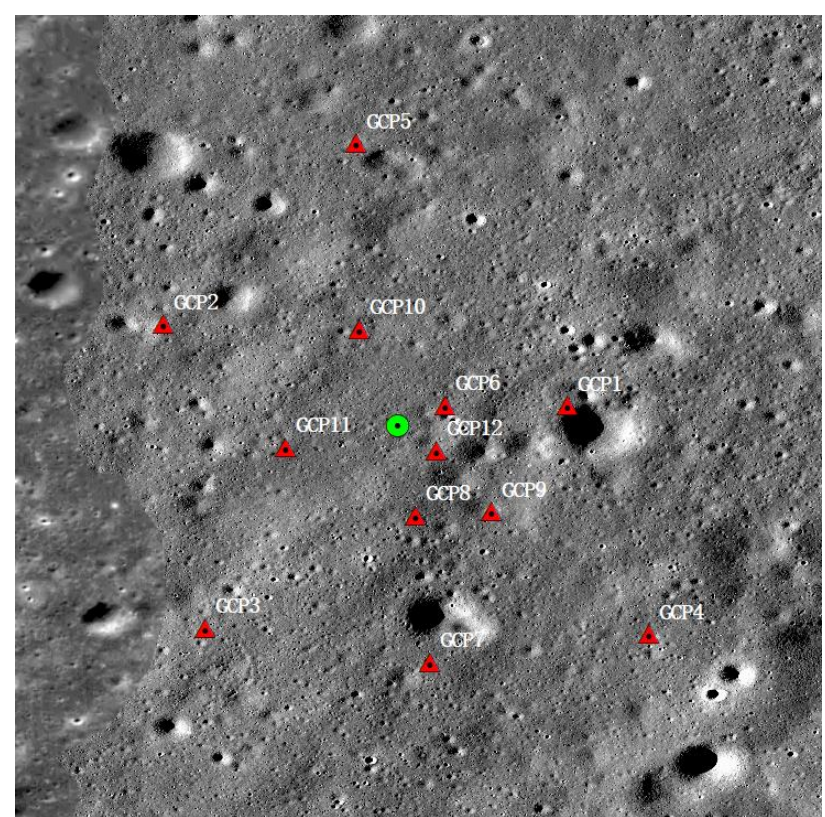

Figure 5. The distribution of selected GCPs

The Cartesian coordinates of the selected GCPs are list below:

\begin{tabular}{|c|c|c|c|}
\hline ID & $\mathrm{X}(\mathrm{m})$ & $\mathrm{Y}(\mathrm{m})$ & $\mathrm{Z}(\mathrm{m})$ \\
\hline GCP1 & -1213615.382 & 50188.14207 & -1233952.96 \\
\hline GCP2 & -1213976.197 & 52426.88323 & -1233501.18 \\
\hline GCP3 & -1212290.074 & 52124.46692 & -1235169.07 \\
\hline GCP4 & -1212357.821 & 49684.19634 & -1235200.66 \\
\hline GCP5 & -1213587.19 & 50858.46344 & -1233943.26 \\
\hline GCP6 & -1212154.433 & 50882.31916 & -1235354.34 \\
\hline GCP7 & -1212965.837 & 50997.65333 & -1234551.81 \\
\hline GCP8 & -1212992.783 & 50578.8684 & -1234516.52 \\
\hline GCP9 & -1213989.576 & 51347.05065 & -1233523.93 \\
\hline GCP10 & -1213315.896 & 51722.33642 & -1234171.83 \\
\hline GCP11 & -1213333.254 & 50897.38801 & -1234189.99 \\
\hline \multicolumn{4}{|c}{ Table 1. The Cartesian coordinates of GCPs }
\end{tabular}

Descent camera of Chang'e-4 mission is similar to that of Chang'e-3 mission. The proposed IOPs of Chang'e- 3 descent camera were employed to the self-calibration based motion estimation as initial values. Iterative least square solution was adopt to calculate accurate EOPs and IOPs of descent images. Table 2 shows the $2 \mathrm{D}$ re-projection errors and $3 \mathrm{D}$ measurement errors of GCPs.

\begin{tabular}{|c|c|c|}
\hline ID & 3D Error $(\mathrm{m})$ & 2D Error (pixel) \\
\hline GCP1 & 2.751296 & 0.294789 \\
\hline GCP2 & 2.766094 & 0.324088 \\
\hline GCP3 & 4.960207 & 0.401836 \\
\hline GCP4 & 8.39764 & 0.205661 \\
\hline GCP5 & 1.492362 & 0.660431 \\
\hline GCP6 & 9.566349 & 0.235382 \\
\hline GCP7 & 6.515824 & 0.361456 \\
\hline GCP8 & 1.868449 & 0.336095 \\
\hline GCP9 & 4.905919 & 0.264975 \\
\hline
\end{tabular}

\begin{tabular}{|l|l|l|}
\hline GCP10 & 9.732207 & 0.210446 \\
\hline GCP11 & 2.242095 & 0.386086 \\
\hline
\end{tabular}

Table 2 . The accuracy of self-calibration solution

The mean errors of 3D measurement and $2 \mathrm{D}$ re-projection were $5.02 \mathrm{~m}$ and 0.33 pixels. Figure 6 shows the recovered trajectory of descent sequential images. The highest altitude off the landing point in this trajectory was $5262 \mathrm{~m}$. It can be seen from this figure that lander adjusted its attitude for the purpose of obstacle avoidance at the height of $2 \mathrm{~km}$ and $100 \mathrm{~m}$.

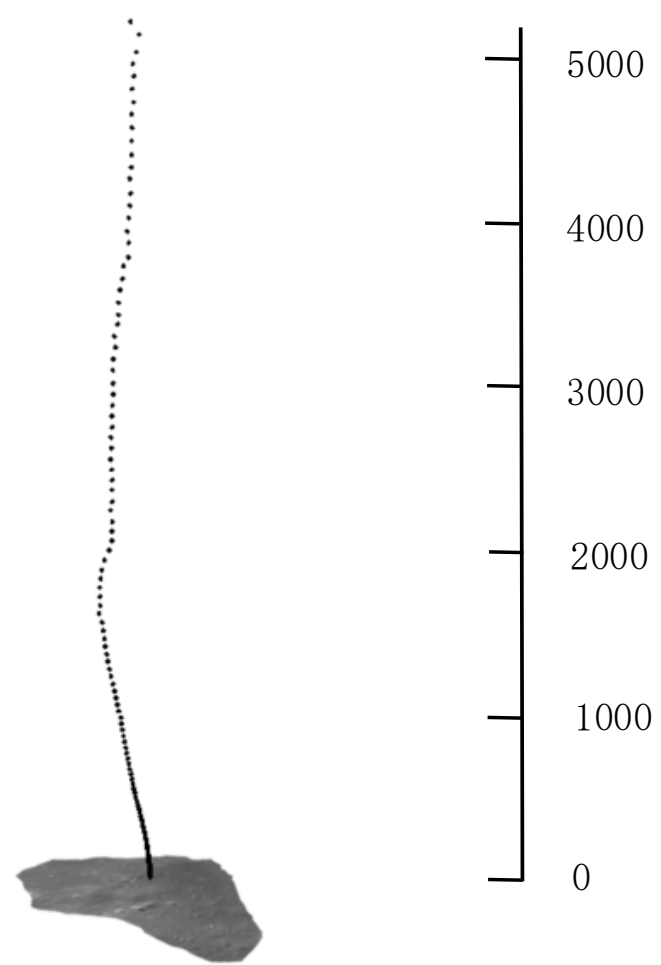

Figure 6 . The recovered trajectory of Chang'e-4 lander

\section{CONCLUSION}

This paper proposed a bundle adjustment based geometric processing method for descent and landing trajectory recovery using descent images. Self-calibration model was employed so as to solve EOPs and IOPs simultaneously with the GCPs selected from the DOM and DEM of landing area generated from LROC NAC image and SLDEM2015. The method and results can support further processing and application of descent images in the future. Meanwhile, we will perform more detailed analysis and comparison about the descent motion and the accuracy of the recovery results with the telemetry data from earth-based control station.

\section{ACKNOWLEDGEMENTS}

This work was supported by the National Natural Science Foundation of China (Grant No. 41671458, 41590851, and 41771488) and the Key Research Program of the Chinese Academy of Sciences (NO. XDPB11). The authors thank all those who worked on the Planetary Data System archive to make the LROC imagery and SLDEM2015 available, and China National Space Administration for providing Chang'e-4 descent images. 


\section{REFERENCES}

Di, K, Jia, M., Xin, X., Liu, B., Peng, M., and Yue, Z., 2018. High resolution seamless DOM generation over Chang'e-5 landing area using LROC NAC images, the Proceedings of 2018 ISPRS TC III Mid-term Symposium "Developments, Technologies and Applications in Remote Sensing, 7-10 May, Beijing, China, 271-276.

Di, K., Liu, Z., Liu, B., Wan, W., Peng M., Wang, Y., Gou, S., Yue, Z., Xin, X., Jia, M., and Niu, S., 2019. Chang'e-4 lander localization based on multi-source data, Journal of Remote Sensing, 23(1):177-184.

Jia, Y., Zou, Y., Xue, C., Ping, J., Yan, J., and Ning, Y., 2018. Scientific objectives and payloads of Chang'E-4 mission. Journal of Space Science, 38(1): 118-130.

Liu, Z., Di, K., Peng, M., Wan, W., Liu, B., Li, L., Yu, T., Wang, B., Zhou, J. and Chen, H., 2015. High precision landing site mapping and rover localization for Chang'e-3 mission. Science China-physics Mechanics \& Astronomy, 58(1): 1-11.

Wan, W., Liu, Z., Liu, Y., Liu, B., Di, K., Zhou, J., Wang, B., Liu, C., and Wang, J., 2014.Descent Image Matching Based Position Evaluation for Chang'e-3 Landing Point. Spacecraft Engineering, 23(4): 5-12. 